\title{
MANIPULATING DRIVE CHARACTERISTICS TO STUDY THE EFFECTS OF MENTAL LOAD ON OLDER AND YOUNGER DRIVERS
}

\author{
Lana M. Trick, Martin Lochner, Ryan Toxopeus, and David Wilson \\ Dept. of Psychology, University of Guelph \\ Guelph, Ontario, Canada \\ Email:1trick@uoguelph.ca
}

\begin{abstract}
Summary: A driving simulator was used to assess performance in younger and older drivers ( $\mathrm{M}$ ages 18 and 71 years). The impacts of three challenges were assessed: visibility (clear day, fog), traffic density (low, high) and wayfinding (no challenge, drivers challenged to use signs and landmarks to find their destination). Performance was measured in terms of hazard RT, collisions, wayfinding errors (missed or extra turns), and driving speed. The challenge manipulations produced interactive effects and age was a factor in some of these interactions. Older drivers missed more turns in wayfinding but overall they performed as well or better than younger drivers and reduced their speed more to driving challenges.
\end{abstract}

\section{INTRODUCTION}

Older drivers are disproportionately at risk for multi-vehicle crashes that occur at places such as intersections: collisions that occur when important information is not picked up (e.g., McGwin \& Brown, 1999; Preusser et al. 1998). In this study we look at different factors that may contribute to crash risk in older drivers, examining three types of potential sources of workload within a drive: visibility challenges, traffic density challenges, and wayfinding challenges.

The research on mental workload owes much to Shiffrin and Schneider (1977). They proposed that though there are many types of mental process (sensory analysis, decision-making, response production, storage and retrieval in memory), all fall into two broad categories. Automatic processes occur without awareness or intent. These processes are effortless and fast, and can be carried out concurrently with other processes without compromising performance: They produce little "load". In contrast, controlled processes occur with awareness, and are deliberate and goal oriented. These are conscious processes and they are also effortful and slow and it is difficult to carry out several controlled processes at once. When two controlled processes are carried out at the same time, there is interference, which is to say, performance is worse on one or both tasks when the tasks are performed together than when they are performed separately. According to this theory, the reason for this interference is that controlled processes share a common limited capacity resource. Each task demands a certain amount of this resource, exacting a certain "load", and if the demands of the two tasks together exceed the capacity of this limited resource, then performance on one or both tasks suffers. For certain controlled processes, extensive practice will make the process automatic, but this can only happen when the process involves a fixed response for a given sensory input. Since this early work, the idea that there is single limited capacity resource has been challenged (e.g., Wickens, 2002) but idea that individuals experience various levels of load depending on characteristics of the task remains. 
Although workload can be measured with questionnaires and physiological measures (e.g., Verwey \& Veltman, 1996), the most common way to assess objective changes in driver performance as a function of load is to use the dual task paradigm. There have been a variety of studies that compare older and younger adults in terms of the amount of interference produced by carrying out secondary tasks. Often these studies look at how adding secondary tasks interferes with driving. For example, there are a number of studies that investigate the effects of various types of conversation on driving in older drivers (e.g., Shinar, Tractinsky, \& Compton, 2005). Others investigate the interference produced by using an in-vehicle device while driving in older drivers (e.g Pohlmann \& Traenkle, 1994). The converse approach involves manipulating the complexity of the drive and measuring the effects on secondary task performance (e.g. Makishita \& Matsunaga, 2008; Verwey, 2000). Overall, though some studies reveal more notable age differences than others, many show more dual-task interference in older than younger drivers. A meta-analysis of the effects of age on dual-task performance suggests that age-related impairments are more pronounced when tasks require substantial amounts of controlled processing (Riby Perfect, \& Stollery, 2004).

The dual task methodology is useful but it has drawbacks. Typical dual task studies require novel combinations of tasks: putting together tasks that typically do not go together, at least for older drivers. For example, a number of studies use mental arithmetic as their chosen secondary task. This task generates substantial amounts of interference (e.g. Makishita \& Matsunaga, 2008; Shinar, Tractinsky, \& Compton, 2005) but most people do not carry out mathematical calculations while driving. Consequently, when older drivers show more interference between tasks, it is unclear whether it is because older drivers have fewer resources for controlled processing or because older drivers have special difficulties with novel combinations of tasks. To avoid this problem, we exploit the fact that the driving task itself may involve different types of load due to various driving challenges: visibility challenges, traffic density challenges, wayfinding challenges. Furthermore, by manipulating these three types of driving challenge, it becomes possible to test Baldwin's (2002) sensory-cognitive interaction theory, which suggests that the deficits in sensory processing serve to accentuate the effects of cognitive demands in older adults. An implication of this theory is that the various types of challenge should produce interactive effects, and these interactions should be especially notable among older drivers.

Three aspects of the drive were manipulated. The first, a visibility manipulation, involved comparing driving in fog with driving in clear weather. The traffic density manipulation involved driving in high or low traffic and the wayfinding manipulation involved comparing performance when drivers simply had to follow the road to arrive at their destination (there were turns but no choice points) to conditions where drivers had to remember the directions and then follow them to arrive at the appropriate destination. To assess the impact of these manipulations we measured hazard RT and collisions, and in the wayfinding challenge condition we also measured wayfinding errors (the number of turns missed due to a failure to follow the directions and respond to the signs and landmarks, the number of extraneous turns). Driving speed was also measured as an index of compensatory behaviour. The primary hypothesis was that the effects of sensory factors (poor visibility) should exaggerate the effects of the other challenges (heavy traffic, wayfinding challenge) on driving performance, especially for older drivers. 


\section{METHODS}

\section{Participants}

Older drivers were healthy active seniors recruited from a seniors' recreational centre and the younger drivers were recruited from the participant pool at University of Guelph. Before the study began, the older adults were first given the Mini-Mental State Exam (MMSE) to ensure they were capable of giving informed consent (all were), and then both young and older drivers were given the Simulator Sickness Questionnaire to identify individuals who were high-risk for simulator adaptation syndrome. High-risk candidates were encouraged to withdraw from the study whereas low-risk candidates continued on for a 10-minute screening test in the simulator, where they had to experience turning and braking. If participants indicated any discomfort, they were withdrawn from the study. This resulted in the loss of 8 young 15 older adults (representing a $28 \%$ and $44 \%$ loss respectively). After screening there were 21 young and 19 older drivers left with mean ages of 18.2 and 70.8 years respectively ( $S D=1.09$ and 5.98 years; 9 and 8 females). All filled out driving history questionnaires. Older adults also completed the Early Treatment of Diabetic Retinopathy Scale (ETDRS), Pelli-Robson Contrast Sensitivity scale (PR), and the Useful Field of View (UFOV) test, specifically the Perceptual speed (PS), Selective Attention (SA) and Divided Attention (DA) subscales. See Table 1.

Table 1. Mean Driver Characteristics for each age group (SD in parentheses)

\begin{tabular}{|l|l|l|l|l|l|}
\hline Drivers & $\begin{array}{l}\text { Km/day } \\
\text { driving }\end{array}$ & $\begin{array}{l}\text { Min/day } \\
\text { driving }\end{array}$ & MMSE & Vision & UFOV \\
\hline $\begin{array}{l}\text { Young } \\
(18 \text { years })\end{array}$ & $\begin{array}{l}46.7 \\
(28.1)\end{array}$ & $\begin{array}{l}59.4 \\
(40.1)\end{array}$ & & & \\
\hline $\begin{array}{l}\text { Older } \\
\text { (71 years })\end{array}$ & 68.7 & $63.6(40.9)$ & 28.7 & ETDRS: $10(.11)$ & PS: 41.6 (66.6) \\
& $(143.6)$ & & $(1.1)$ & PR: $1.45(.07)$ & SA:106.4(106.7) \\
& & & DA: $189.2(80.3)$ \\
\hline
\end{tabular}

\section{Apparatus and Driving Scenario}

A DriveSafety DS-600c simulator was used for testing: a 4-door sedan surrounded by 6 wraparound screens to provide a $300^{\circ}$ degree virtual driving environment. Each simulated drive involved traversing a standard 2-lane highway with a posted speed limit of $90 \mathrm{kph}$. Three factors were manipulated to assess the influence of load in the drive. Visibility was manipulated by comparing driving on a clear day (items always visible within the line of sight) with driving in simulated fog (objects obscured if more than $600 \mathrm{~m}$ away). Traffic density was manipulated by comparing performance when there was roughly 1 car per every 1500 meters (low density) to 1 every 150 meters (high density). Finally, the wayfinding challenge was manipulated by comparing performance when participants simply had to follow the road to arrive at their destination (drivers made turns but there were no choice points in the drive, and no need to pay attention to sign posts or landmarks) to that when drivers had to remember the name of the destination town and follow the sign and use a landmark to arrive at their destination. This involved the 8 choice points, 6 of which were distractor choice points where no turn was required. To encompass this $2 \times 2 \times 2$ design, there were eight -15 minute drives that were carried out on two consecutive days for the older drivers. (Testing took longer for older drivers 
because they did a number of psychological tests.) The order in which drivers experienced the different drives was counterbalanced. In each drive, there were periodic hazards. Dogs, cyclists or vehicles intruded into the vehicle's path.

Hazard RT was calculated from the time when the hazard first entered the drivers' lane and when the driver braked, and collisions were defined as the number of times that the drivers collided with the simulated entities in the vehicle. Finally, wayfinding errors were defined as the number of missed directions (failures to turn at the appropriate sign or landmark) and the number of extra turns that the drivers made that were not in response to directions. Lane keeping ( $S D$ of lane position) was also measured but it is not reported in this paper due to length restrictions.

\section{RESULTS}

For each of the four dependent variables, factorial split plot analyses of variance were carried out with age as the sole between subjects factor. Hazard RT were marginally lower for older adults $\left(F(1,38)=3.56, p=.067, \eta^{2}=.09\right)$. Both visibility and traffic density had significant effects $\left(F(1,38)=5.12, p=.029, \eta^{2}=.12\right.$ and $\left.F(1,38)=11.44, p=.002, \eta^{2}=.23\right)$ but the wayfinding manipulation did not $(F<1)$. There was a significant Age X Visibility interaction $(F(1,38)=$ $\left.7.08, p=.011, \eta^{2}=.16\right)$ insofar as the fog had a bigger effect on hazard RT for younger adults (Figure 1). A Visibility X Traffic Density X Wayfinding interaction emerged $(F((1,38)=22.06$, $\left.p<.001,, \eta^{2}=.37\right)$, such that fog had a bigger effect in low-density traffic than high when there was no wayfinding challenge, but there was no four-way interaction with age as a factor $(p>.1)$.

Hazart RT: Younger adults

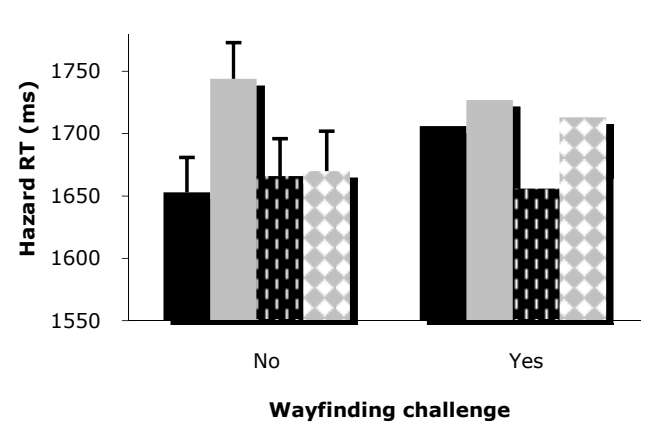

Hazard RT: Older adults

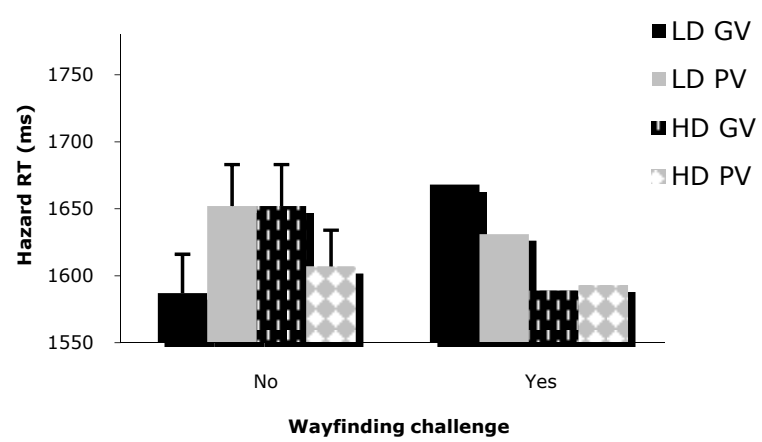

Figure 1. Hazard RT in older and younger drivers: $L D=$ Low Density Traffic; HD = High Density Traffic; GV = Good Visibility; PV = Poor Visibility (Fog). SE bars included

Collisions were relatively rare, but nonetheless, younger drivers had significantly more than older drivers $\left(F(1,38)=4.62, p=.04, \eta^{2}=.11\right)$, with averages of 0.21 and .05 per driver respectively over the 8 drives (Figure 2$)$. There was also a four-way interaction: Age $\mathrm{X}$ Visibility X Density X Wayfinding challenge $\left(F(1,38)=4.50, p=.04, \eta^{2}=.11\right)$, such that the younger drivers were especially at risk when visibility was good and traffic density low but they had to follow signs and landmarks to get to their destination. 
Total collisions: Younger adults

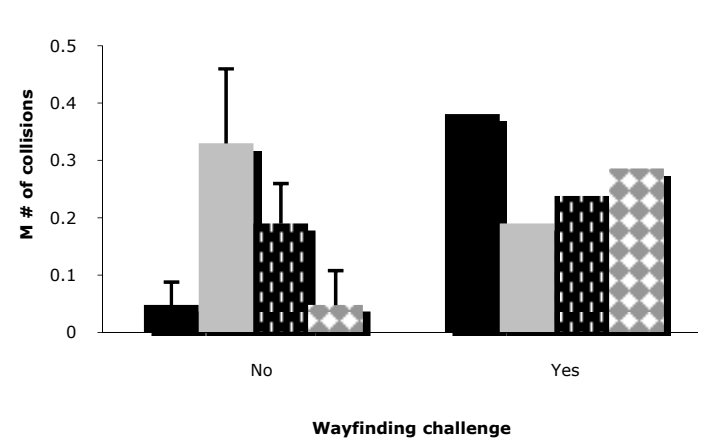

Total collisions: Older adults

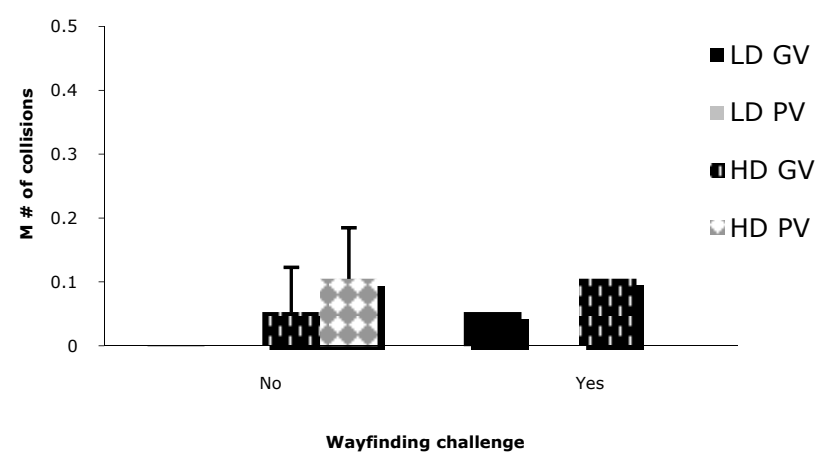

Figure 2. Collisions in older and younger drivers: LD = Low Density Traffic; HD = High Density Traffic; GV = Good Visibility; PV = Poor Visibility (Fog). SE bars included

For each wayfinding challenge drive, participants had to turn in response to a sign and a landmark. Thus the maximum number of missed turns per drive was two for each of the four drives. Older adults missed significantly more turns $\left(F(1,38)=5.66, p=.022, \eta^{2}=.13\right)$. The average number of missed turns was .47 in the older adults and .23 for younger adults. For both groups, there was no significant difference in the number of missed signs as compared to landmarks, or the number of missed first versus second turns ( $F<1$ for both). Interestingly, for both groups, participants were more likely to miss turns under good visibility conditions than poor $\left(F(1,38)=11.33, p=.002, \eta^{2}=.23\right)$. The number of excess turns was unaffected by age group or any of the other factors $(\mathrm{F}<1)$. See Figure 3.

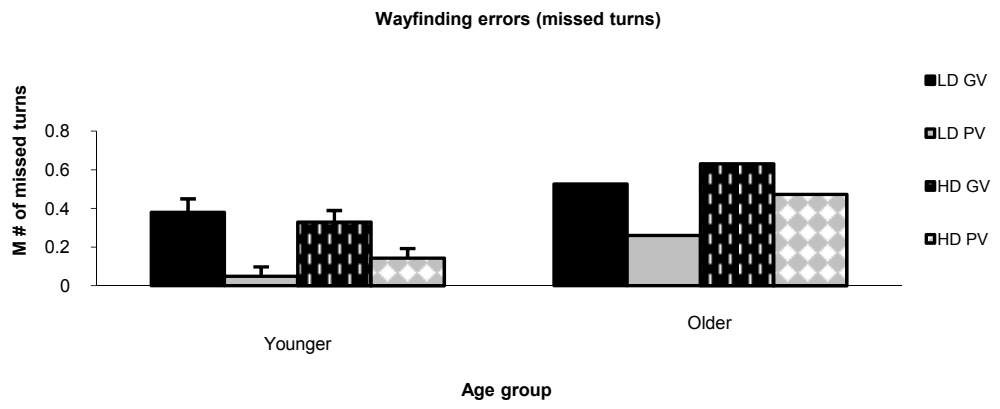

Figure 3. Number of missed turns in older and younger drivers: $L D=$ Low Density Traffic; HD $=$ High Density Traffic; GV = Good Visibility; PV = Poor Visibility (Fog). SE bars included

Older drivers had lower driving speeds $\left(F(1,38)=18.35, p<.001, \eta^{2}=.33\right)$. Driving speeds were also lower in fog $\left(F(1,38)=11.81, p=.001, \eta^{2}=.24\right)$, high-density traffic $(F(1,38)=$ $\left.18.66, p<.001, \eta^{2}=.33\right)$ and when there were wayfinding challenges $(F(1,38)=62.32, p<$ $\left..001, \eta^{2}=.62\right)$. All three variables interacted with age, which suggests that older drivers reduce their speed more in reaction to each of the three challenges (Age X Visibility $(F(1,38)=13.02, p$ $<.001, \eta^{2}=.26$; Age X Density: $F(1,38)=4.16, p=.049, \eta^{2}=.10 ;$ Age X Wayfinding $(F(1,38)$ $\left.=24.55, p<.001, \eta^{2}=.39\right)$. See Figure 4 . 
Driving speed: Younger adults

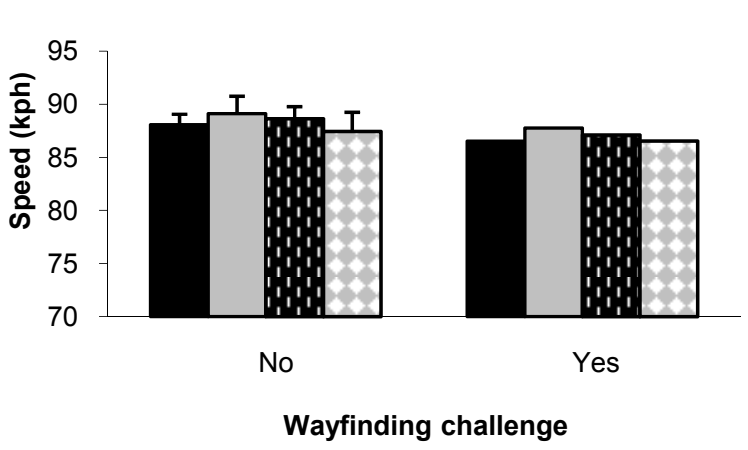

Driving speed: Older adults

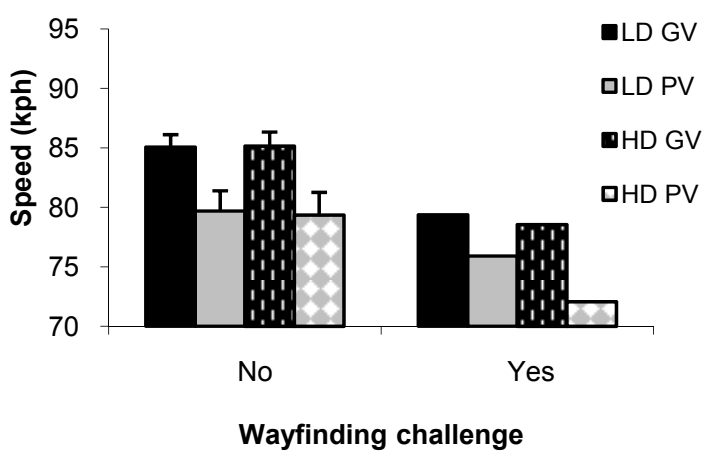

Figure 4. Driving speed in older and younger drivers: $L D=$ Low Density Traffic; HD = High Density Traffic; GV = Good Visibility; PV = Poor Visibility (Fog). SE bars included

\section{DISCUSSION}

There were two main findings. First, contrary to what might be expected from typical dual task studies, in this study when the load of the drive was increased by adding together different types of driving challenge, in all save the wayfinding task, the performance of older drivers was as good or better than that of younger drivers. This may be because older adults adjusted their speed more in response to the driving challenges. The second finding relates to Baldwin's sensory cognitive interaction theory (2002), which predicts sensory deficits (limitations in visibility) should accentuate the effects of other types of cognitive demand (traffic density, wayfinding). This theory suggests there should be three-way interactions between challenge factors (Visibility $\mathrm{X}$ Density X Wayfinding) and four-way interactions involving age (Age X Visibility X Density $\mathrm{X}$ Wayfinding) because reduced visibility should be especially deleterious for older adults given age-related sensory deficits. Three and four-way interactions emerged in the hazard RT and collision data respectively, but interactions did not take the form expected. For example, younger adults were especially at risk of collision when visibility was good, traffic density was low, and they had to find their way using directions, perhaps because they were driving about as fast as they did with no wayfinding challenge. This highlights the importance of measuring compensatory behaviours when assessing the impact of sensory and cognitive demands.

Although the results of this study are interesting it is possible that they are more indicative of the detrimental effects of inexperience than the benign effects of age. In this study the younger drivers were all within 5 years of obtaining their license ( $M=1.9$ years). Moreover, compared to the older drivers, the young drivers reported fewer kilometers and less time per day driving. Fatigue may have also been a factor in the poor performance of younger drivers because they were tested in one 2-hour session, unlike the older drivers. It is possible that the older adults in this study were especially good drivers insofar as they chose to come to the university to be tested. As well, because a disproportionate number of older drivers did not make it through the screening for simulator sickness ( $44 \%$ of the older drivers had to be dropped as compared to $28 \%$ of the younger drivers) it is possible that the ones that remained were especially robust and fit. Nonetheless, overall this study calls into question the idea that older adults necessarily perform worse than others in the face of the various challenges of the driving task. 


\section{ACKNOWLEDGEMENTS}

The Ontario Neurotrauma Fund, the Canadian Foundation for Innovation, the Ontario Innovation Trust, and Auto21 funded this research. Lauren Meegan helped with testing participants.

\section{REFERENCES}

Baldwin, C.L. (2002). Designing in-vehicle technologies for older drivers: Applications of sensory-cognitive interaction theory. Theoretical Issues in Ergonomic Science, 3(4), 307329.

Makishita, H., \& Matsunaga, K. (2008). Differences of drivers' reactions times according to age and mental workload. Accident Analysis and Prevention, 40, 567-575.

McGwin, G., \& Brown, D. (1999). Characteristics of traffic crashes among young, middle-aged, and older drivers. Accident Analysis and Prevention, 31(1), 181-189.

Pohlmann, S., \& Traenkle, U. (1994). Orientation in road traffic. Age-related differences in using an in-vehicle navigation system and a conventional map. Accident Analysis and Prevention, $26,687-702$.

Preusser, D., Williams, A., Ferguson, S., Ulmen, R., \& Weinstein, H. (1998). Fatal crash risk for older drivers at intersections. Accident Analysis and Prevention, 30(2), 151-159.

Riby, L.M., Perfect, T.J., \& Stollery, B.T. (2004). The effects of age and task domain on dual task performance: A meta-analysis. European Journal of Cognitive Psychology, 16(6), 863891.

Shiffrin, R. and Schneider, W. (1977), Controlled and automatic human information processing: II. Perceptual learning, automatic attending, and a general theory, Psychological Review, 84, 127-190.

Shinar, D., Tractinsky, N., \& Compton, R. (2005). Effects of practice, age, and task demands, on interference from a phone task while driving. Accident Analysis and Prevention, 37, 315-326

Verwey, W.B. (2000). On-line driver workload estimation. Effects of road situation and age on secondary task measures. Ergonomics, 43(2), 187-209.

Verwey, W.B., \& Veltman, H.A. (1996). Detecting short periods of elevated workload: A comparison of nine workload assessment techniques. Journal of Experimental Psychology: Applied. 2(3), 270-285.

Wickens, C. (2002). Multiple resources and performance prediction. Theoretical Issues in Ergonomic Science, 3, 159-177. 